\title{
ARTICLE
}

\section{Dynamic kirigami structures for integrated solar tracking}

\author{
Aaron Lamoureux ${ }^{1}$, Kyusang Lee ${ }^{2}$, Matthew Shlian ${ }^{3}$, Stephen R. Forrest ${ }^{1,2} \&$ Max Shtein ${ }^{1,3}$
}

Optical tracking is often combined with conventional flat panel solar cells to maximize electrical power generation over the course of a day. However, conventional trackers are complex and often require costly and cumbersome structural components to support system weight. Here we use kirigami (the art of paper cutting) to realize novel solar cells where tracking is integral to the structure at the substrate level. Specifically, an elegant cut pattern is made in thin-film gallium arsenide solar cells, which are then stretched to produce an array of tilted surface elements which can be controlled to within $\pm 1^{\circ}$. We analyze the combined optical and mechanical properties of the tracking system, and demonstrate a mechanically robust system with optical tracking efficiencies matching conventional trackers. This design suggests a pathway towards enabling new applications for solar tracking, as well as inspiring a broader range of optoelectronic and mechanical devices.

\footnotetext{
${ }^{1}$ Department of Materials Science and Engineering, University of Michigan, Ann Arbor, Michigan 48109, USA. ${ }^{2}$ Department of Electrical Engineering and Computer Science, University of Michigan, Ann Arbor, Michigan 48109, USA. ${ }^{3}$ School of Art and Design, University of Michigan, Ann Arbor, Michigan 48109, USA. Correspondence and requests for materials should be addressed to S.R.F. (email: stevefor@umich.edu) or to M.S. (email: mshtein@umich.edu).
} 
C onventional photovoltaic modules suffer optical coupling losses due to a decrease in projected area that scales with the cosine of the misalignment angle between the cell and the sun (Fig. 1a). To mitigate these losses and maximize power output, flat photovoltaic panels can be tilted to track the position of the sun over the course of the day and/or year. Depending on the geographic location of the system, and whether there are one or two tracking axes, conventional trackers can provide an increase in yearly energy generation between 20 and $40 \%$ compared with non-tracking solar arrays ${ }^{1,2}$. Furthermore, tracking may be integrated with concentrated photovoltaic systems, where source alignment is critical for maintaining a high concentration factor over a wide range of source angles ${ }^{3-11}$.

Despite the documented effectiveness and relatively mature state of solar tracking, such systems have not been widely implemented due to the high costs, added weight, and additional space required to align the panels, support panel weight, and resist wind loading ${ }^{12,13}$. For example, current data indicate that the additional components required for tracking account for $\sim 12 \%$ of the total balance of system costs, a number that is increasing at $\sim 1 \%$ per year ${ }^{14}$. This is in contrast to solar cell and module costs, which continue to decrease rapidly ${ }^{15}$. Furthermore, because of the cumbersome nature of conventional tracking mechanisms, their use has thus far been limited to ground-based and flat-rooftop installations. As a result, residential, pitched rooftop systems, which account for $\sim 85 \%$ of installations, lack conventional tracking options entirely ${ }^{14,16}$. To further decrease installation costs and enable new applications, a novel approach to compact and lightweight solar tracking is required.

The principles of origami and kirigami (that is, the folding and cutting of paper, respectively, to achieve a desired shape) have been used in the design of airbags, optical components, stowable spaceborne solar arrays, reprogrammable metamaterials and load-bearing metal structures ${ }^{17-21}$. Here, utilizing similar design principles, we show for the first time simple, dynamic kirigami structures integrated with thin-film solar cells that enable highly efficient and macroscopically planar solar tracking as a function of uniaxial strain. For optimized systems, we show tracking to within $\pm 1.0^{\circ}$ of the predicted pointing vector, with total power generation approaching that of conventional singleaxis tracking systems. Kirigami trackers are also shown to be

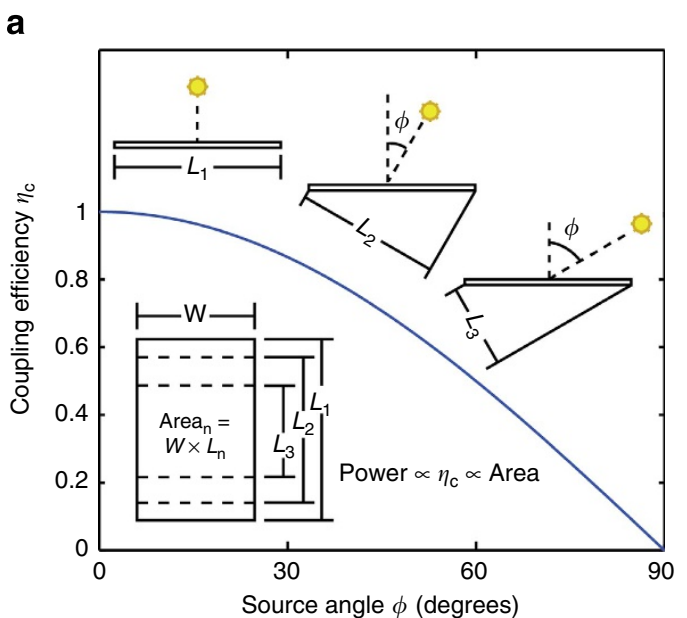

electrically and mechanically robust, with no appreciable decrease in performance after $>300$ cycles.

\section{Results}

Kirigami design principles for integrated solar tracking. Consider the simple kirigami structure in Fig. 1b, consisting of a linearly cut pattern in an otherwise thin, continuous sheet of flexible material. Pulling on the sheet in the axial direction (that is, perpendicular to the cuts) results in instabilities that produce controlled buckling in the transverse direction (that is, parallel to the cuts), along with a change in feature angle that is synchronized along its length. Importantly, it is possible to control the direction of the feature tilt (that is, clockwise or counter-clockwise with respect to the original plane) by lifting or lowering one end of the sheet before the straining process (Fig. 1c). By integrating similar structures with thin-film solar cells, this unique geometric response may be used as a novel method to accurately track the sun. In practice, these thin-film trackers may be housed inside of thin, double-pane enclosures to ensure weatherproofing and provide support against sagging at larger length scales (Supplementary Fig. 1). To enable dual-axis tracking, the kirigami tracker need simply be rotated about an axis normal to its plane (Supplementary Fig. 2).

The geometric response of a simple kirigami structure is clarified for a Kapton sheet tracker in Fig. 2a, where the kirigami geometry is defined by the cut length $\left(L_{\mathrm{C}}\right)$, as well as the spacing between cuts in the transverse $(x)$ and axial $(y)$ directions. The change in feature angle $(\theta)$ and decrease in sample width (that is, transverse strain, $\varepsilon_{\mathrm{T}}$ ) as a function of stretching (that is, axial strain, $\varepsilon_{\mathrm{A}}$ ) are:

$$
\begin{gathered}
\theta=\cos ^{-1}\left(\frac{1}{\varepsilon_{\mathrm{A}}+1}\right) \\
\varepsilon_{\mathrm{T}}=\frac{R_{1}-1}{R_{1}+1}\left[\cos \left(\sin ^{-1}\left(\frac{2 R_{1} \tan \theta}{R_{1} R_{2}-R_{2}}\right)\right)-1\right]
\end{gathered}
$$

where $\varepsilon_{\mathrm{A}}=\frac{L-L_{0}}{L_{0}}, \varepsilon_{\mathrm{T}}=\frac{W-W_{0}}{W_{0}}$ and $R_{1}=\frac{L_{\mathrm{C}}}{x}$ and $R_{2}=\frac{L_{\mathrm{C}}}{y}$ are dimensionless parameters (Supplementary Note 1). To examine the effect of system geometry on response, $R_{1}$ and $R_{2}$ were systematically varied such that $R_{1}=R_{2}=3,5,10$ and 20 , as shown in Fig. 2b. The response characterized by equations (1)

\section{b}

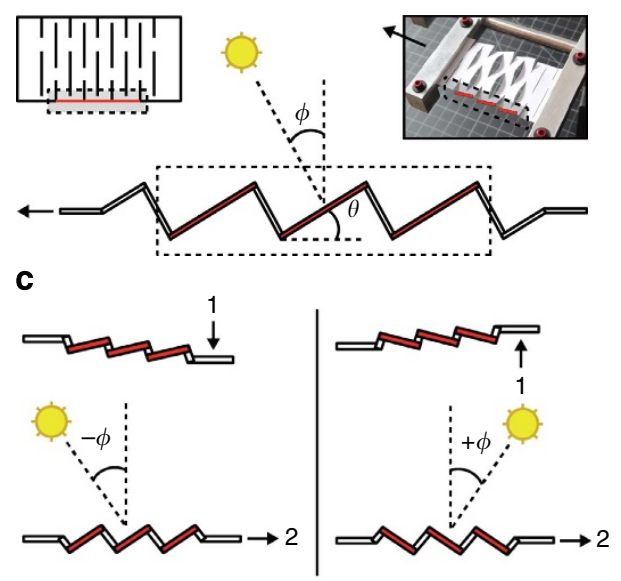

Figure 1 | Optical coupling efficiency and novel kirigami trackers. (a) Coupling efficiency $\left(\eta_{C}\right)$ versus source angle $(\phi)$ for a planar solar panel. The panel projected area decreases with the $\cos \phi$. (b) A kirigami tracking structure that, upon stretching, simultaneously changes the angle of the elements comprising the sheet. By incorporating thin-film solar cells into this structure, it may be used as a low-profile alternative to conventional single-axis solar tracking. (c) The direction of feature tilt (that is, clockwise or counter-clockwise with respect to the original plane) is controlled by lifting or lowering one end of the sheet (step 1) before the straining process (step 2). 
a
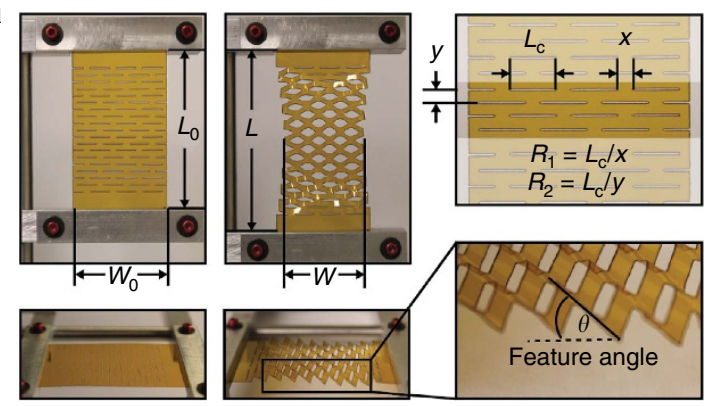

b

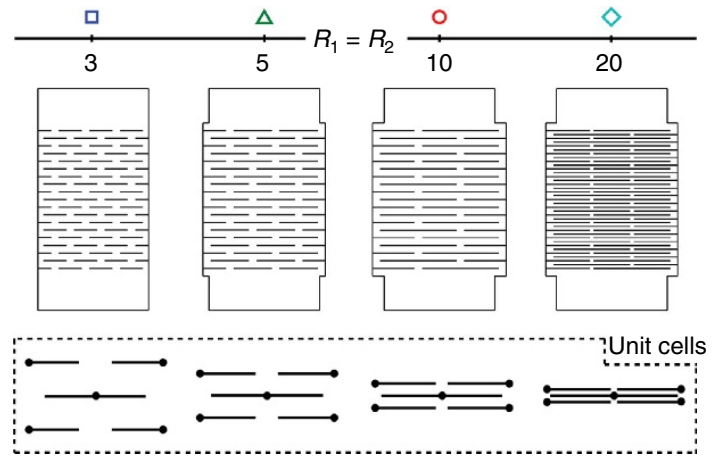

c

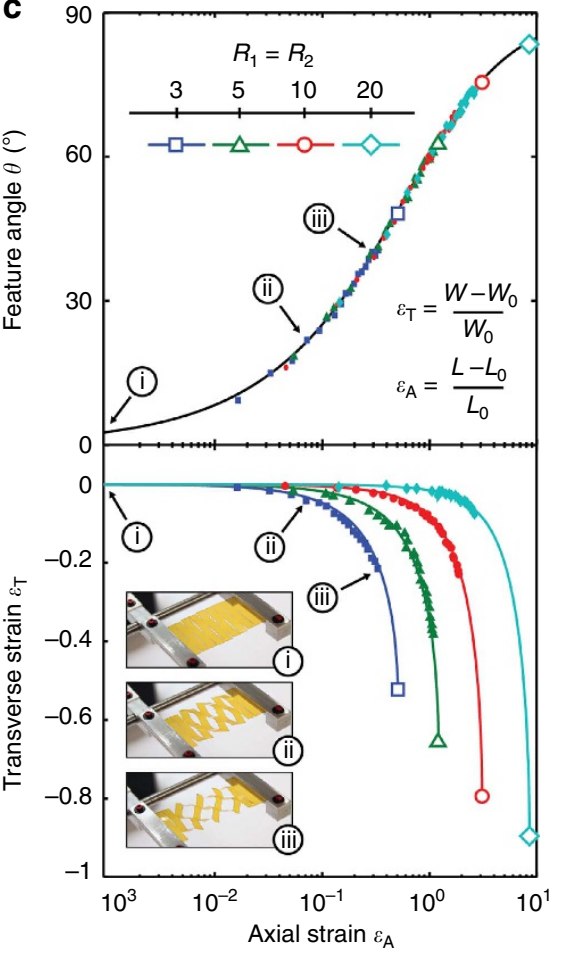

Figure 2 | Kirigami cut geometry and geometric system response. (a) Response of a Kapton kirigami structure to stretching in the axial direction ( $\varepsilon_{\mathrm{A}}$ ) is accompanied by a decrease in sample width $\left(\varepsilon_{T}\right)$ and a change in feature angle $(\theta)$. Also shown are the geometric parameters that define the kirigami structure, namely the cut length $\left(L_{C}\right)$ and spacing between cuts in the transverse $(x)$ and axial (y) directions, which can be expressed in terms of the dimensionless parameters, $R_{1}$ and $R_{2}$. (b) Schematics of four kirigami structures, where $R_{1}=R_{2}=3,5$, 10 and 20, along with their corresponding units cells. (c) $\varepsilon_{\mathrm{T}}$ and $\theta$ versus $\varepsilon_{\mathrm{A}}$ for several kirigami structures where $R_{1}=R_{2}=3,5,10$ and 20 (b). Theoretical predictions per equations (1) and (2) are shown by solid lines, while the closed symbols represent experimental data from a $50 \mu \mathrm{m}$-thick Kapton sample of the appropriate geometry. While larger $R_{1}$ and $R_{2}$ enable increased axial strains and correspondingly larger transverse strains, the change in feature angle is independent of cut geometry.

and (2) (solid lines) is experimentally verified (closed symbols) in Fig. 2c for each kirigami structure. We find that larger $R_{1}$ and $R_{2}$ enable greater $\varepsilon_{\mathrm{A}}$ and correspondingly greater $\varepsilon_{\mathrm{T}}$. A plot of $\theta$ versus $\varepsilon_{\mathrm{A}}$ confirms that the dependence of $\theta$ on $\varepsilon_{\mathrm{A}}$ is independent of cut geometry. For the samples tested, $\theta$ was controlled to within $\pm 1.0^{\circ}$ of its value in equation (1). Also shown are the pseudo-plastic limits for each superstructure, where the maximum axial strains and corresponding maximum feature angles, $\theta_{\mathrm{MAX}}$, are depicted as open symbols. For each structure, $\theta_{\mathrm{MAX}}$ is solely dependent on cut geometry, setting the upper limit of tracking without shadowing in the axial direction. That is:

$$
\theta_{\mathrm{MAX}}=\tan ^{-1}\left(\frac{R_{1} R_{2}-R_{2}}{2 R_{1}}\right)
$$

Optical properties and optimized tracking performance. Remarkably, this simple structure is very effective for solar tracking, which we quantify as follows. We define the total optical coupling efficiency $\left(\eta_{\mathrm{C}}\right)$ as a function of individual optical coupling losses in the axial $\left(\eta_{\mathrm{A}}\right)$ and transverse $\left(\eta_{\mathrm{T}}\right)$ directions, as well as cosine losses $\left(\eta_{\mathrm{O}}\right)$ and surface reflection $\left(\eta_{\mathrm{R}}\right)$ at oblique incident angles. For an optical source moving in the plane normal to the tracking axis, and assuming a suitable anti-reflective coating, $\eta_{\mathrm{C}}$ is simply:

$$
\eta_{\mathrm{C}}=\cos \phi\left(\varepsilon_{\mathrm{A}}+1\right)\left(\varepsilon_{\mathrm{T}}+1\right)
$$

where $\phi$ is the source angle from the normal to the module plane (Fig. 1a), and $\varepsilon_{\mathrm{A}}$ and $\varepsilon_{\mathrm{T}}$ are the axial and transverse strains, respectively (Supplementary Note 2 ). To maximize $\eta_{\mathrm{C}}$, shadowing in the axial direction, as well as cosine losses, must be balanced against the decrease in the width resulting from the geometric response of the structure. Indeed, tracking to $\theta_{\text {MAX }}$ may not be optimal due to the sharp decrease in projected area beyond some critical strain (Fig. 2c). This geometric subtlety is quantified in Fig. 3a, where we analyze the differences in performance for optimized tracking (open symbols), and one variation of non-optimized tracking to $\theta_{\mathrm{MAX}}$ defined by equation (3) (closed symbols).

As shown in the inset of Fig. 3a $(\theta$ versus $\phi)$, each structure tracks the source as characterized by a unity slope, until a predetermined value of $\theta^{*}$ is reached (that is, extent of tracking), after which the angle of the structure is held constant. Here, $\theta^{*}$ is denoted as point 1 and point 2 for non-optimized and optimized tracking, respectively. The effects of these tracking modes are shown in the plot of $\eta_{C}$ versus $\phi$, as defined by equation (4). Note the difference in $\eta_{\mathrm{C}}$ at large values of $\phi$. Whereas tracking to $\theta_{\text {MAX }}$ (closed symbols) causes a large decrease in sample width and $\eta_{\mathrm{C}}$ near the geometric limits of the structure, optimized tracking (open symbols) minimizes the tradeoff between sample narrowing $\left(\eta_{\mathrm{T}}\right)$, self-shadowing $\left(\eta_{\mathrm{A}}\right)$ and cosine losses $\left(\eta_{\mathrm{O}}\right)$. Figure $3 \mathrm{~b}$ shows the extension of this analysis to other cut geometries, where $\eta_{\mathrm{C}}$ is integrated over a range of tracking angles (from $\phi=0$ to $\phi=\theta^{*}$ ) and normalized to conventional planar cell performance under identical operating conditions. Figure $3 \mathrm{~b}$ provides the appropriate tracking procedure for a given kirigami structure, where optimal performance is obtained by tracking the source at normal incidence until reaching the $\theta^{*}$ corresponding to the maximum of each curve (for $R_{1}=R_{2}=3, \theta^{*} \approx 37^{\circ}$, for $R_{1}=R_{2}=5, \theta^{*} \approx 54^{\circ}$, for $R_{1}=R_{2}=10, \theta^{\star} \approx 73^{\circ}$, and for 

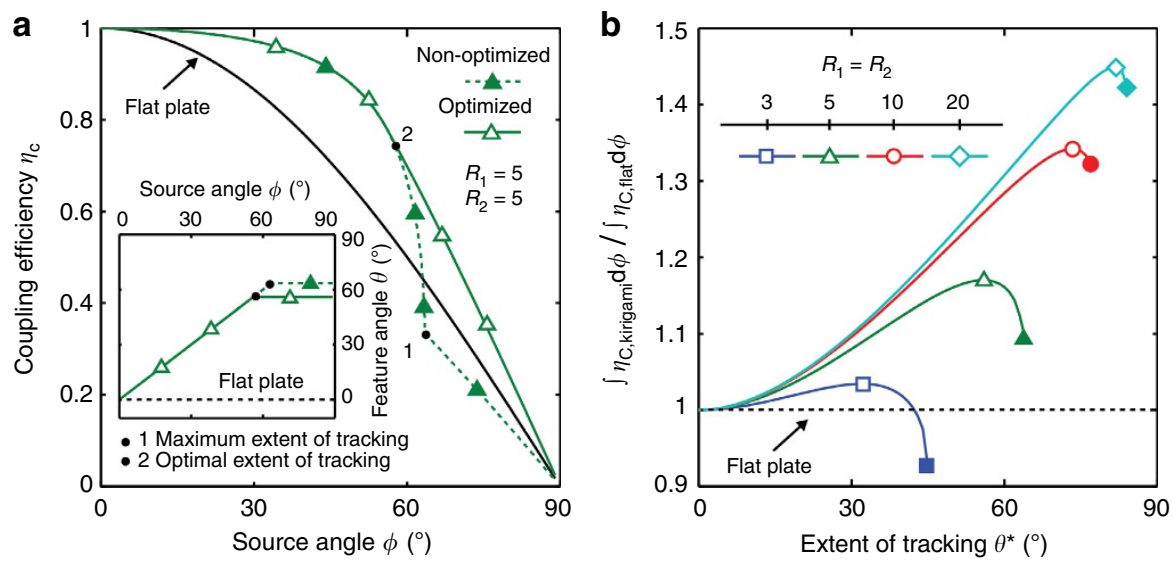

Figure 3 | Optimization of tracking process to maximize coupling efficiency. (a) Coupling efficiency $\left(\eta_{C}\right)$ versus source angle $(\phi)$ for two systems with different extent of tracking $\left(\theta^{\star}\right)$. Inset: Feature angle $(\theta)$ versus $\phi$. Non-optimized tracking (closed symbols) close to the geometric maximum ( $\theta_{\mathrm{MAX}}$, point 1) results in a sharp decrease in sample width that decreases optical coupling efficiency. Instead, coupling efficiency is optimized (open symbols) by a tradeoff between sample narrowing, self-shadowing, and cosine losses (c.f. equation (4) in text) corresponding instead to an optimal angle (point 2). Simulated system response is shown for $R_{1}=R_{2}=5$, and is compared to a conventional non-tracking panel. (b) Coupling efficiency, $\eta_{C}$ integrated over a range of tracking angle (from $\phi=0$ to $\phi=\theta^{\star}$ ) and normalized to conventional planar cell performance. For a given kirigami structure, optimal performance is obtained by tracking the source at normal incidence to $\theta^{\star}$ corresponding to the maximum of each curve. For comparison, tracking to $\theta_{\mathrm{MAx}}$ versus tracking to the optimal $\theta^{*}$ is shown as solid and open symbols, respectively.

$R_{1}=R_{2}=20, \theta^{\star} \approx 82^{\circ}$ ). For comparison, tracking to $\theta_{\mathrm{MAX}}$ versus the optimized tracking limit are shown as solid and open symbols, respectively.

Fabrication of GaAs kirigami tracker. Due to the buckling of the sheet along the transverse direction, it is advantageous to combine this tracking design with solar cells that can similarly flex to accommodate the non-planarity of the sheet. Hence, we mounted thin $(\sim 3 \mu \mathrm{m})$, flexible, crystalline gallium arsenide (GaAs) photovoltaic cells with the kirigami tracking structure in Fig. 2. The cells were fabricated by a combination of non-destructive (and thus potentially low cost) epitaxial lift-off (ELO) and cold welding between gold films pre-deposited on both the backside of the GaAs solar cells and on the Kapton structure using procedures described previously ${ }^{22-24}$. Notably, the solar cells were patterned to coincide with predetermined values of $R_{1}$ and $R_{2}$ to minimize edge recombination losses as well as prevent damage during the laser dicing cell singulation process. Finally, a bilayer anti-reflective coating consisting of $\mathrm{TiO}_{2}$ and $\mathrm{MgF}_{2}$ was used to minimize reflection losses at oblique angle incidence resulting from the bowing of the kirigami cells during stretching (Supplementary Fig. 3).

Validation of tracking performance. An example kirigami tracker with $>99 \%$ GaAs solar cell coverage is shown in Fig. 4a, where $R_{1}=R_{2}=3$. Each tracker was systematically strained to follow a moving AM1.5G collimated light source, and the solar cell current density versus voltage $(J-V)$ characteristics were obtained as a function of illumination angle, $\phi$. A schematic of this experiment is shown in the inset of Fig. $4 \mathrm{~b}$, where the kirigami tracker was strained to track the light source to the optimal $\theta^{\star}$ per the process shown in Fig. 3. Figure $4 \mathrm{~b}$ plots the ratio of the normalized angle-dependent short circuit current density $\left(J_{\mathrm{SC}}(\phi) / J_{\mathrm{SC}}(\phi=0)\right)$ for two samples, where $R_{1}=R_{2}=3$ and $R_{1}=R_{2}=5$ (closed symbols). Also shown is $\eta_{\mathrm{C}}$ defined by equation (4) for several cut geometries (open symbols, solid lines). As expected, larger $R_{1}$ and $R_{2}$ lead to an increase in $\eta_{\mathrm{C}}$ due to the suppression of $\varepsilon_{\mathrm{T}}$ at equivalent $\varepsilon_{\mathrm{A}}$. Furthermore, $J_{\mathrm{SC}}(\phi) / J_{\mathrm{SC}}(\phi=0)$ matches $\eta_{\mathrm{C}}$ predicted by equation (4), suggesting that $\eta_{C}$ is a direct measure of optical coupling in the presence of a suitable cell anti-reflective coating.

The effect of kirigami geometry on power generation is compared with fixed planar cells and conventional single-axis systems in Fig. 4c for Phoenix, AZ $\left(33.45^{\circ} \mathrm{N}, 112.07^{\circ} \mathrm{W}\right)$ during the summer solstice. The power conversion efficiency for each system was assumed to be $20 \%$. As $R_{1}$ and $R_{2}$ increase, the system becomes more efficient in the morning and evening at angles far from the zenith, and the output power density increases accordingly. As shown in the inset, the output energy density for an optimized kirigami system approaches that of conventional single-axis tracking in the limit of large values of $R_{1}$ and $R_{2}$. This is indeed remarkable for a solar cell that remains essentially flat and without a change in macroscopic orientation. For more information on system response (that is, $\theta$ and $\eta_{C}$ versus time of day for $R_{1}=R_{2}=10$ ), see Supplementary Fig. 4 .

Effect of mechanical stresses on device performance. The electrical and mechanical responses to strain and cycling were also considered with implications for long-term solar tracking. For the Kapton/GaAs tracker where $R_{1}=R_{2}=5$, there was no systematic change in either fill factor $(F F)$ or open circuit voltage $\left(V_{\mathrm{OC}}\right)$ from $\theta=0$ to $\theta^{*}$ (Supplementary Fig. $5 \mathrm{a}$ ), with repeated measurements over 350 cycles yielding similar results (Supplementary Fig. 5b). Over a similar range, strain energy (that is, the energy stored in the system undergoing deformation) was shown to decrease by $\sim 36 \%$, without failure, due to plastic deformation and crack propagation at the cuts for high strains. By optimizing the cut geometry and thus minimizing stress at the cuts, it is possible to significantly decrease strain fade-for example, in comparable Kapton kirigami trackers where $R_{1}=R_{2}=3,5$ and 10 , the strain energy was shown to decrease by $\sim 74, \sim 17$ and only $\sim 3 \%$, respectively, over 1,000 cycles. Other substrates with improved mechanical and thermal stabilities (for example, spring steel) are also currently being investigated as more robust materials platforms with longer operational lifetimes.

\section{Discussion}

In summary, we have shown that kirigami structures combined with thin-film active materials may be used as a simple, low-cost, 
$\mathbf{a}$
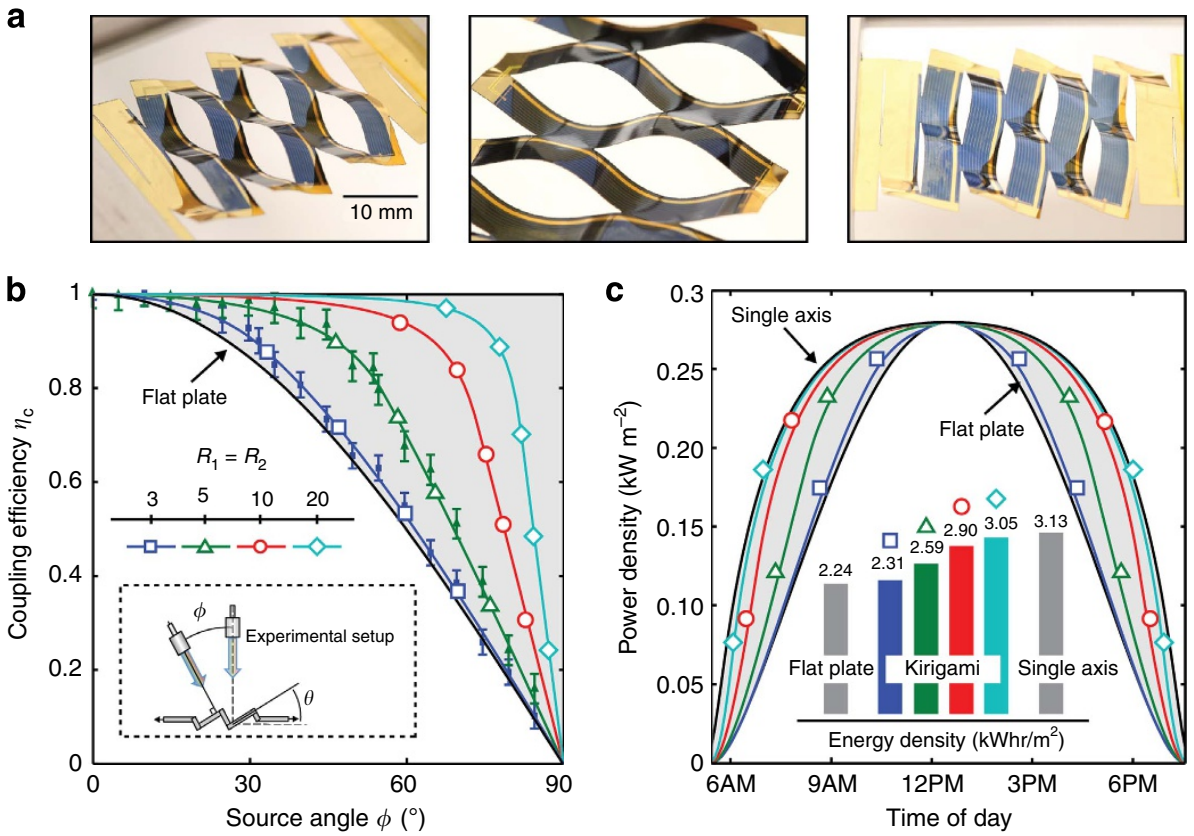

Figure 4 | Tracking performance for GaAs kirigami trackers. (a) Integrated thin-film, crystalline GaAs solar cells, mounted by cold weld bonding on a Kapton carrier substrate, as used for testing. Here, $L_{C}=15 \mathrm{~mm}, x=5 \mathrm{~mm}$ and $y=5 \mathrm{~mm}\left(R_{1}=R_{2}=3\right)$. (b) Normalized solar cell short circuit current density $J_{\mathrm{SC}}(\phi) / J_{\mathrm{SC}}(\phi=0)$ for two samples, where $R_{1}=R_{2}=3$ and $R_{1}=R_{2}=5$ (closed symbols). Also shown are the simulated data for coupling efficiency $\left(\eta_{C}\right)$ obtained from equation (4) (solid lines, open symbols). The agreement between experimental and simulated results suggests that $\eta_{C}$ is a direct measure of optical coupling, and that performance may be optimized by increasing $R_{1}$ and $R_{2}$. (c) Output electrical power density incident on the solar cell versus time of day for several kirigami cut structures, stationary panel and single-axis tracking systems in Phoenix, $\mathrm{AZ}\left(33.45^{\circ} \mathrm{N}, 112.07^{\circ} \mathrm{W}\right.$ ) during the summer solstice. Inset: Integration of the curves yields the associated energy densities, where kirigami-enabled tracking systems are capable of near single-axis performance.

lightweight and low-profile method to track solar position, thereby maximizing solar power generation. These systems provide benefits over conventional mechanisms, where additional heavy, bulky components and structural supports are often required to synchronize tracking between panels and accommodate forces due to wind loading. By eliminating the need for such components, kirigami serves to decrease installation costs and expose new markets for solar tracking, including widespread rooftop, mobile and spaceborne installations. Kirigami-enabled systems are also cost-effective and scalable in both fabrication and materials, and similar design rules may be extended for use in a wide range of optical and mechanical applications, including phased array radar and optical beam steering.

\footnotetext{
Methods

Fabrication of thin-film GaAs solar cells. Epitaxial layers of $p-n$ junction gallium arsenide (GaAs) active material on an AlAs sacrificial layer were grown by gas-source molecular beam epitaxy on a 2-inch diameter (100) GaAs substrate. For the ND-ELO process, $0.2 \mu \mathrm{m}$ thick GaAs buffer layer followed by a $20-\mathrm{nm}$ thick AlAs sacrificial layer were grown, first. Then, following inverted photovoltaic device layers were grown: $0.1 \mu \mathrm{m}$ thick, $5 \times 10^{18} \mathrm{~cm}^{-3}$ Be-doped GaAs $p$-contact layer, $0.025 \mu \mathrm{m}$ thick, $2 \times 10^{18} \mathrm{~cm}^{-3}$ Be-doped $\mathrm{Al}_{0.20} \mathrm{In}_{0.49} \mathrm{Ga}_{0.31} \mathrm{P}$ window layer, $0.15 \mu \mathrm{m}$ thick, $1 \times 10^{18} \mathrm{~cm}^{-3}$ Be-doped $p$-GaAs emitter layer, $3.0 \mu \mathrm{m}$ thick, $2 \times 10^{17} \mathrm{~cm}^{-3}$ Si-doped $n$-GaAs base layer, $0.05 \mu \mathrm{m}$ thick, $6 \times 10^{17} \mathrm{~cm}^{-3}$ Si-doped $\operatorname{In}_{0.49} \mathrm{Ga}_{0.51} \mathrm{P}$ back surface field layer, and $0.05 \mu \mathrm{m}$ thick, $5 \times 10^{18} \mathrm{~cm}^{-3}$ Si-doped $n$-GaAs contact layer. The sample was then coated with a $300 \mathrm{~nm}$ thick $\mathrm{Au}$ layer by e-beam evaporation, and bonded to a $50 \mu \mathrm{m}$-thick E-type Kapton sheet (also coated in $300 \mathrm{~nm}$ Au layer) using cold weld bonding by applying a pressure of $4 \mathrm{MPa}$ for $3 \mathrm{~min}$ at a temperature of $200^{\circ} \mathrm{C}$. After bonding, the photovoltaic epitaxial active region and Kapton carrier were isolated from the bulk wafer using ELO by selectively removing the AlAs sacrificial layer in dilute (15\%) hydrofluoric acid (HF) solution at room temperature. After ND-ELO, a $\mathrm{Pd}(5 \mathrm{~nm}) / \mathrm{Zn}(20 \mathrm{~nm}) /$ $\mathrm{Au}(700 \mathrm{~nm})$ front metal contact was patterned using photolithography. Then, the device mesas were similarly defined using photolithography and subsequent
}

chemical etching using $\mathrm{H}_{3} \mathrm{PO}_{4}: \mathrm{H}_{2} \mathrm{O}_{2}$ :deionized $\mathrm{H}_{2} \mathrm{O}$ (3:1:25). The exposed, highly Be-doped $150 \mathrm{~nm}$ thick $p+$ GaAs contact layer was selectively removed using plasma etching. After annealing the sample for $1 \mathrm{~h}$ at $200^{\circ} \mathrm{C}$ to facilitate ohmic contact formation, the sidewalls were passivated with $1-\mu \mathrm{m}$ thick polyimide applied by spin coating. After curing the sample at $300^{\circ} \mathrm{C}$ for $30 \mathrm{~min}$, the polyimide was selectively removed by photolithography and plasma etching. The external contact pad was patterned with $\mathrm{Ti}(10 \mathrm{~nm}) / \mathrm{Au}(500 \mathrm{~nm})$. Finally, a bilayer anti-reflection coating consisting of $\mathrm{TiO}_{2}(49 \mathrm{~nm})$ and $\mathrm{MgF}_{2}(81 \mathrm{~nm})$ was deposited by e-beam evaporation.

Fabrication of kirigami trackers. Kapton kirigami structures were fabricated using a $50 \mathrm{~W}$ Universal Laser Systems $\mathrm{CO}_{2}$ laser (2\% power, $2.5 \%$ speed, 500 pulses per inch (PPI)). Following the schematics in Fig. $2 b$, the following cut dimensions were used; $R_{1}=R_{2}=3\left(L_{\mathrm{C}}=6 \mathrm{~mm}, x=2 \mathrm{~mm}\right.$ and $\left.y=2 \mathrm{~mm}\right), R_{1}=R_{2}=5$ ( $L_{\mathrm{C}}=10 \mathrm{~mm}, x=2 \mathrm{~mm}$ and $\left.y=2 \mathrm{~mm}\right), R_{1}=R_{2}=10\left(L_{\mathrm{C}}=20 \mathrm{~mm}, x=2 \mathrm{~mm}\right.$ and $y=2 \mathrm{~mm}), R_{1}=R_{2}=20\left(L_{\mathrm{C}}=20 \mathrm{~mm}, x=1 \mathrm{~mm}\right.$ and $\left.y=1 \mathrm{~mm}\right)$. Kapton $/ \mathrm{GaAs}$ solar trackers were fabricated using the ND-ELO process described previously, and then cut using an X-ACTO knife. As outlined in the main text, two samples were used, namely $R_{1}=R_{2}=3\left(L_{\mathrm{C}}=15 \mathrm{~mm}, x=5 \mathrm{~mm}\right.$ and $\left.y=5 \mathrm{~mm}\right)$ and $R_{1}=R_{2}=5$ $\left(L_{C}=15 \mathrm{~mm}, x=3 \mathrm{~mm}\right.$ and $\left.y=3 \mathrm{~mm}\right)$. It should be noted that, for the cycling $J-V$ measurements, the GaAs was patterned only along the dimension $x$ (Supplementary Note 2) to eliminate the effect of $\varepsilon_{\mathrm{T}}$ and $\eta_{\mathrm{C}}$ on electrical response (that is, to ensure a constant $J_{\mathrm{SC}}$ and comparable $J-V$ curves).

Measurement of axial and transverse strain. The Kapton kirigami structures described previously were systematically strained to the maximum feature angle, $\theta_{\operatorname{MAX}}$ (equation (3)), using a homemade micro-strain apparatus. The straining process was imaged in situ using two cameras: one facing directly downwards to capture $\varepsilon_{\mathrm{T}}$ and a second facing the edge of the sample to capture $\theta$ (Fig. 2a). Both cameras captured the axial strain, $\varepsilon_{\mathrm{A}}$. The resulting images were analysed using ImageJ (W.S. Rasband, US National Institutes of Health, Bethesda, Maryland, USA), where a global calibration scale was used to define measurement lengths. It should be noted that, in some cases, limitations imposed by the range of motion of the apparatus prohibited data collection at high strain values, as shown in Fig. 2c.

Electrical characterization of GaAs kirigami trackers. The Kapton/GaAs kirigami trackers described previously were systematically strained using a 
micro-strain apparatus to track a moving AM1.5G light source (Oriel solar simulator, model 91191 with Xenon arc lamp and AM 1.5 global filter, simulated 1 sun, $100 \mathrm{~mW} \mathrm{~cm}^{-2}$ intensity), following the optimal tracking process described in Fig. 3 (that is, $\theta=0^{\circ}$ to $\theta=\theta^{\star}$ ). The $J-V$ characteristics (that is, $J_{\mathrm{SC}}, V_{\mathrm{OC}}$ and FF) were measured at each angle using a semiconductor parameter analyser (SPA, Agilent 4155B), in increments of five degrees, from normal incidence $\left(\phi=0^{\circ}\right)$ to $\phi=90^{\circ}$. To determine the effect of cycling on cell performance, the solar tracker was repeatedly strained to $\theta=\theta^{*}$, while the source was kept constant at $\phi=0^{\circ}$. Cell performance (that is, $J_{\mathrm{SC}}, V_{\mathrm{OC}}$ and $F F$ ) was measured every 10 cycles, when $\theta=0$ (and consequently $\phi=0^{\circ}$ ), for 350 cycles (to simulate $\sim 1$ year of operation), as shown in Fig. 4 d.

\section{Mechanical characterization of kirigami trackers. The stress-strain} characteristics of the Kapton and Kapton/GaAs kirigami trackers described previously were measured using a TA.XTPlus Texture Analyser (Texture Technologies, Hamilton, Massachusetts, USA) and the Exponent (Texture Technologies, Hamilton, Massachusetts, USA) software package. For the Kapton trackers, the sample length as measured from the first cut to the last cut in the axial direction was $36 \mathrm{~mm}$. For the Kapton/GaAs trackers, the sample length as measured from the first cut to the last cut in the axial direction was $33 \mathrm{~mm}$. Each sample was strained per the optimized tracking process described in Fig. 3, and the resulting stress-strain behaviour was recorded. This process was repeated 1,000 times, and the resulting curves were integrated to find the strain energy. The strain fade was calculated as the percentage difference in strain energy between cycle 1 and cycle 1,000 (that is, $100 \% \times \frac{E_{1}-E_{1,000}}{E_{1}}$ ).

\section{References}

1. Mousazadeh, H. et al. A review of principle and sun-tracking methods for maximizing solar systems output. Renew. Sust. Energ. Rev. 13, 1800-1818 (2009).

2. Lee, C. Y., Chou, P. C., Chiang, C. M. \& Lin, C. F. Sun tracking systems: a review. Sensors 9, 3875-3890 (2009).

3. Feuermann, D. \& Gordon, J. High-concentration photovoltaic designs based on miniature parabolic dishes. Sol. Energy 70, 423-430 (2001).

4. Sierra, C. \& Vazquez, A. J. High solar energy concentration with a Fresnel lens. J. Mater. 40, 1339-1343 (2005).

5. Ryu, K., Rhee, J., Park, K. \& Kim, J. Concept and design of modular Fresnel lenses for concentration solar PV system. Sol. Energy 80, 1580-1587 (2006).

6. Currie, M. J., Mapel, J. K., Heidel, T. D., Goffri, S. \& Baldo, M. A. Highefficiency organic solar concentrators for photovoltaics. Science 321, 226-228 (2008).

7. Yoon, J. et al. Flexible concentrator photovoltaics based on microscale silicon solar cells embedded in luminescent waveguides. Nat. Commun. 2, 1-8 (2011).

8. Duerr, F., Meuret, Y. \& Thienpont, H. Tracking integration in concentrating photovoltaics using laterally moving optics. Opt. Express 19, A207-A218 (2011).

9. Sheng, X. et al. Doubling the power output of bifacial thin-film GaAs solar cells by embedding them in luminescent waveguides. Adv. Energy Mater. 3, 991-996 (2013).

10. Duerr, F., Meuret, Y. \& Thienpont, H. Tailored free-form optics with movement to integrate tracking in concentration photovoltaics. Opt. Express 21, A401-A411 (2013).

11. Price, J. S., Sheng, X., Meulblok, B. M., Rogers, J. A. \& Giebink, N. C. Wide-angle planar microtracking for quasi-static microcell concentrating photovoltaics. Nat. Commun. 6, 1-8 (2015).

12. Bhaduri, S. \& Murphy, L. M.Solar Energy Research InstituteUnited States Department of Energy. Wind Loading on Solar Collectors (Solar Energy Research Institute, 1985).

13. GE Energy. Western Wind and Solar Integration Study. NREL/SR-550-47434 (2010).

14. Goodrich, A., James, T. \& Woodhouse, M. Residential, Commercial, and Utilityscale Photovoltaic (PV) System Prices in the United States: Current Drivers and Cost-reduction Opportunities (U.S. Department of Energy, 2012).

15. U.S. Department of Energy. Sunshot Vision Study DOE/GO-102012-3037 (2012)
16. Barbose, G. Tracking the Sun VI: An Historical Summary of the Installed Price of Photovoltaics in the United States from 1998 to 2012. Report No. LBNL-6350E, (Lawrence Berkeley National Laboratory, Berkeley, 2014.

17. Mroz, K. \& Pipkorn, B. in 6th European LS-DYNA User's Conference (Gothenburg, Sweden, 2007).

18. Cho, J. H. et al. Nanoscale origami for 3D optics. Small 7, 1943-1948 (2011).

19. Zirbel, S. et al. Accommodating thickness in origami-based deployable arrays. J. Mech. Des 135, 111005 (2013).

20. Silverberg, J. L. et al. Using origami design principles to fold reprogrammable mechanical metamaterials. Science 345, 647-650 (2014).

21. Cruz, P. J. S., Buri, H. U. \& Weinand, Y. in ICSA2010 1st International Conference on Structures \& Architecture (CRC Press/Balkema Taylor \& Francis Group, 2010)

22. Konagai, M., Sugimoto, M. \& Takahashi, K. High efficiency GaAs thin film solar cells by peeled film technology. J. Cryst. Growth 45, 277-280 (1978).

23. Lee, K., Shiu, K., Zimmerman, J. D., Renshaw, C. K. \& Forrest, S. R. Multiple growths of epitaxial lift-off solar cells from a single InP substrate. Appl. Phys. Lett. 97, 101107 (2010)

24. Lee, K., Zimmerman, J. D., Hughes, T. W. \& Forrest, S. R. Non-destructive wafer recycling for low-cost thin-film flexible optoelectronics. Adv. Funct. Mater. 24, 4284 (2014)

\section{Acknowledgements}

We thank Paul Dodd, Chih-Wei Chen and Terry Shyu for discussions; Olga Shalev, Adam Barito, Matt Sykes and Kanika Agrawal for extensive support and comments; and Marv Cressey and Kent Pruss for laser training and support. This work was performed in part at the Laurie Nanofabrication Facility, a member of the National Nanotechnology Infrastructure Network, which is supported in part by the National Science Foundation (NSF). We (A.L., M.Shlian, M.Shtein) acknowledge the partial financial support of the National Science Foundation (NSF), grant NSF 1240264 under the Emerging Frontiers in Research and Innovation (EFRI) programme, (A.L., M.S.) Department of Air Force grant: FA9550-12-1-0435, as well as (K.L. and S.F.) NanoFlex Power Corp.

\section{Author contributions}

A.L. derived the mathematical expressions to model system geometry, carried out experimental set-up and measurements, fabricated kirigami structures and ran simulations to model tracking performance. K.L. fabricated thin-film GaAs solar cells. M.Shlian contributed to fabrication and iteration of initial designs. M.Shtein and S.F. supervised the work. A.L. and M.Shtein originated the study, prepared the manuscript and all authors contributed to data interpretation, discussions and writing.

\section{Additional information}

Supplementary Information accompanies this paper at http://www.nature.com/ naturecommunications

Competing financial interests: The authors declare no competing financial interests.

Reprints and permission information is available online at http://npg.nature.com/ reprintsandpermissions/

How to cite this article: Lamoureux, A. et al. Dynamic kirigami structures for integrated solar tracking. Nat. Commun. 6:8092 doi: 10.1038/ncomms9092 (2015).

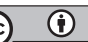

This work is licensed under a Creative Commons Attribution 4.0 International License. The images or other third party material in this article are included in the article's Creative Commons license, unless indicated otherwise in the credit line; if the material is not included under the Creative Commons license, users will need to obtain permission from the license holder to reproduce the material. To view a copy of this license, visit http://creativecommons.org/licenses/by/4.0/ 